\title{
The Spreading Width Calculation of Giant Resonances with a Semi-Microscopic Approach
}

\author{
T. N. Leite ${ }^{1,2}$ and N. Teruya ${ }^{2}$ \\ 1 Colegiado de Engenharia Civil \\ Fund. Universidade Federal do Vale do São Francisco \\ C.P. 309, 48900-000 Juazeiro, BA, Brazil. \\ 2 Departamento de Física, Universidade Federal da Paraíba \\ C.P. 5008, 58051-970 João Pessoa, PB, Brazil
}

Received on 26 August, 2006

\begin{abstract}
We have proposed a semi-microscopic approach to calculate the two particles - two holes $(2 p-2 h)$ spreading width of giant resonances. Our proposal has been based in a hybrid method that implements the statistical multistep compound theory of Feshbach, Kerman and Koonin $(F K K)$, widely and successful used in nuclear reactions mechanisms, in order to include relevant informations about the microscopic structure obtained by the Random Phase Approximation ( $R P A)$ calculations. This method is an approximative calculation to avoid the intrinsic numerical difficulties of those microscopic calculations that incorporate more complex structure than one particle - one hole $(1 p-1 h)$ excitations. Unlike the reaction context, the residual interaction was adjusted in $R P A$ calculation to reproduce the lowest energy levels of the studied nuclei. The feasibility and the efficiency of the approach has been tested in giant dipole resonances in ${ }^{208} \mathrm{~Pb}$ and neutron-rich calcium isotopes, ${ }^{48} \mathrm{Ca}$ and ${ }^{60} \mathrm{Ca}$.
\end{abstract}

Keywords: Giant Resonance, Statistical Multistep Compound Theory, Random Phase Approximation, Spreading Width.

\section{INTRODUCTION}

The calculation of the decay widths of the giant resonances $(G R)$ in nuclei supplies a measure of the fragmentation degree of their decay mechanisms. These mechanisms have taken a special attention in nuclear physics research $[1,2]$ since the discovered of those highly collective nuclear excitations. The $G R$ 's energy is located above the threshold of particle emission and its classification in terms of the direct and statistical decay modes has been a permanent discussion in the literature. The competition between these decay modes can be formulated through an evolution chain in a particle-hole basis, which allows the decomposition of the total width $(\Gamma)$ in two components: the escape width $\left(\Gamma^{\uparrow}\right)$, that couples directly one particle to the continuum, and the spreading width $\left(\Gamma^{\downarrow}\right)$, due to the coupling to internal degrees of freedom like lowlying surface modes and $2 p-2 h$ excitations. In this sense, the total width can be written as $\Gamma=\Gamma^{\uparrow}+\Gamma^{\downarrow}$. The study of the competition between these decay modes has always motivated the development of methods of calculations of these widths, bringing new insights to the understanding of the resonances microscopic structure. The Random Phase Approximation $(R P A)$ has been reaching a lot of theoretical success to describe the GR microscopically as a coherent superposition of particle-hole excitations. Various different versions of this theory have been developed to treat the escape process by considering the coupling of $1 p-1 h$ excitations to the continuum [3-10]. Beside these efforts the study of the spreading process also had their improvements by extending the $1 p-1 h$ basis in order to include $2 p-2 h$ excitations [11-13] in a $R P A-$ like treatment, named second $R P A$. However, in face to the enormous dimensions of the matrices that describe the second RPA these calculations become impracticable, mainly when applied to the heavy nuclei, requiring a long time of numerical computation. To outline these intrinsic numerical difficulties some approximated methods were developed to calculate the widths related with more complex structure than $1 p-1 h$ [14-19].

In Ref. [20], we have analysed the structure of the isovector dipole resonance in neutron-rich calcium isotope, ${ }^{60} \mathrm{Ca}$, by using a previous version of the continuum $R P A$ approach [10], which was modified to take into account the differences among the neutron and proton radii in nuclei with neutron excess. We have observed a small escape width of the giant dipole resonance $(G D R)$ in this nucleus, indicating that the more complicated excitations than $1 p-1 h$ should also be important for the description of its microscopic structure. Recently, we have applied the same method for the analyses of the direct decay mode of isoscalar giant dipole resonances (ISGDR) in ${ }^{208} \mathrm{~Pb}$ [21], whose new studies have been stimulating the improvements of the structure calculations largely around the ISGDR [19, 22-27].

The purpose of this paper is to complete the investigation about the $G R$ decay mechanisms, and to present a feasible scheme to calculate the GR's spreading width. Therefore, we present a new formulation of an approximated semi-microscopic method, based in the statistical Multi-Step Compound Theory $(M S C)$ of Feshbach, Kerman and Koonin $(F K K)$ [28] in connection with the RPA calculations. This procedure overcomes the inherent numerical difficulties in the second $R P A$ to investigate the $2 p-2 h$ structure, and also allows to include in the $F K K$ approach the same residual interaction used in the RPA calculations. This fact is an important point regarding the management of the residual interaction, what in our proposal it is made in consonance with the $R P A$ calculations, maintaining a cohesion between both the approaches. This method has been tested in giant dipole resonances in ${ }^{208} \mathrm{~Pb}$ and neutron-rich calcium isotopes, ${ }^{48} \mathrm{Ca}$ and ${ }^{60} \mathrm{Ca}$.

This paper is a more detailed and complete version of the work: "Description of Decay Mechanisms of the Giant Dipole Resonances with a RPA+FKK Approach"; proceedings 
of XXVII Workshop on Nuclear Physics in Brazil (2004), published in special issue of Brazilian Journal of Physics [29].

In the Sect. II of this paper, we present the semimicorscopical approach used in the spreading width calculation. The results are discussed in the Sect. III.

\section{THE SEMI-MICROSCOPIC APPROACH}

In order to perform the calculation of the spreading width, we can use the Statistical Multi-Step Compound Theory $(M S C)$ of Feshbach, Kerman and Koonin $(F K K)$ [28]. In this approach, the excitation of the $G R$ takes place in a number of stages, the particle emission is allowed in anyone of them. Each stage is represented by a level of complexity, which is characterized by the number of particle-hole pairs that are excited by an external field: the first stage has $1 p-1 h$ configuration, the second one has $2 p-2 h$ configuration and so on, until the compound nucleus formation [30].

According to this formalism, the average total width $\left\langle\Gamma_{n J}\right\rangle$ for each stage $n(n p-n h)$ with angular momentum $J$ is given by the sum of the escape contribution $\left\langle\Gamma_{n J}^{\uparrow}\right\rangle$, due to the continuum coupling, and the spreading contribution $\left\langle\Gamma_{n J}^{\downarrow}\right\rangle$, due to the transition for more complex stages. The spreading width for the $n$th stage, for a excitation energy $E$, is given by

$$
\left\langle\Gamma_{n J}^{\downarrow n+1}(E)\right\rangle=2 \pi \frac{\left\langle\left|H_{n, n+1}^{J}\right|^{2}\right\rangle}{\left\langle D_{n+1 J}\right\rangle}
$$

where $\left\langle\left|H_{n, n+1}^{J}\right|^{2}\right\rangle$ is the mean square matrix element of interaction that couples the $n$ and $n+1$ stages with total angular momentum $J$, and $\left\langle D_{n+1 J}\right\rangle$ is the average spacing of levels in the $(n+1)$ st stage coupling to the total angular momentum $J$. The average process is performed over initial states and summed over final states by considering the particle-hole level density $\rho_{p h}(E, J)$ with energy $E$ and angular momentum $J$.

By assuming an energy and angular momentum factorization of the state density $\rho_{p h}(E, J)$,

$$
\rho_{p h}(E, J)=\omega(p, h, E) R_{n}(J),
$$

the spreading width is written as [28]:

$$
\left\langle\Gamma_{n J}^{\downarrow n+1}(E)\right\rangle=X_{n J}^{\downarrow n+1} Y_{n}^{\downarrow n+1}(E)
$$

The $X$ function contains the angular momentum structure included in the particle-particle two-body interaction and the spin distribution of the single particle levels. The $Y$ function contains all the dependence on excitation energy originating from the final state density, the particle-hole distinguishability, and describes the available phase space for the transition.

In order to calculate the $X$ function we are considering the diagrammatic representation for the process with the additional creation of a particle-hole pair (see FIG. 1). By performing the average process with a general two-body interaction, we have obtained

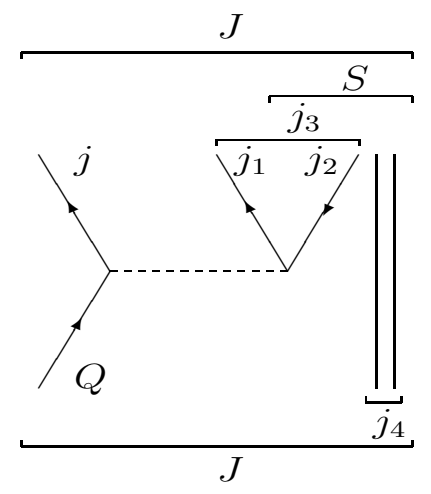

FIG. 1: Diagram with the angular momentum coupling [30] used in the $X$ function computation for the process which one particlehole pair is created. As we do not considered the distinguishability between particle (up arrow) and hole (down arrow), the two-body interaction (dashed line) is particle-particle type.

$$
\begin{aligned}
X_{n J}^{\downarrow(n+1)}= & 2 \pi \sum_{j Q j_{4} j_{3}} \frac{R_{1}(j) R_{1}(Q) R_{N-1}\left(j_{4}\right)}{R_{N}(J)} \frac{\left(2 j_{3}+1\right)}{(2 Q+1)} \Delta\left(Q J j_{4}\right) \\
& \sum_{j_{1} j_{2}} R_{1}\left(j_{1}\right) R_{1}\left(j_{2}\right)\left(\left\langle\left\{j_{1} j_{2}\right\}_{j_{3}}|V|\{Q j\}_{j_{3}}\right\rangle\right)^{2}
\end{aligned}
$$

where

$$
R_{N}(J)=\frac{(2 J+1)}{\pi^{\frac{1}{2}} N^{\frac{3}{2}} \sigma^{3}} \exp \left(-\frac{\left(J+\frac{1}{2}\right)^{2}}{N \sigma^{2}}\right)
$$

is the angular momentum distribution of the single-particle levels with a spin cut-off parameter

$$
\sigma=\left[\frac{\sqrt{12}}{45 \pi} \frac{A^{\frac{5}{3}}}{g}\right]^{\frac{1}{2}}
$$

related to the single-particle spacing $g$, and

$$
N=\left\{\begin{array}{c}
p+h=2 n, N \text { even } \\
p+h+1=2 n+1, N \text { odd }
\end{array}\right.
$$

is the number of the excited particle-hole pairs (number of excitons). The function $\Delta\left(j_{a} j_{b} j_{c}\right)$ guarantees the angular momentum conservation, i.e., $\Delta\left(j_{a} j_{b} j_{c}\right)=1$ if $\left|\vec{j}_{a}-\vec{j}_{b}\right| \leq j_{c} \leq$ $\left|\vec{j}_{a}+\vec{j}_{b}\right|$ or zero otherwise.

The computation of the $Y$ function is similar to that of the $X$. In this case, we have considered the state densities of the initial and final particle-hole configurations. Due to the particlehole distinguishability, there are two spreading process, corresponding to a particle or a hole interacting with a bound nucleon, exciting an additional particle-hole pair (see FIG. 2). So we have

$$
Y_{n}^{\downarrow n+1}(E)={ }_{a} Y_{n}^{\downarrow n+1}(E)+{ }_{b} Y_{n}^{\downarrow n+1}(E)
$$




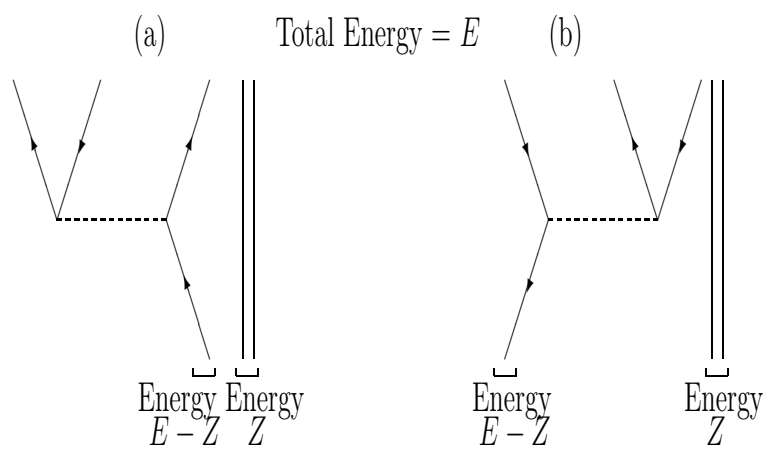

FIG. 2: Diagram with the energy conservation [30] in order to calculate the $Y$ function considering the two possible processes to excite a particle-hole pair, taking account the particle-hole distinguishability.

with

${ }_{a} Y_{n}^{\downarrow n+1}(E)=\int_{0}^{E} \frac{\omega(1,0, E-z) \omega(p-1, h, z)}{\omega(p, h, E)} \omega(2,1, E-z) d z$

${ }_{b} Y_{n}^{\downarrow n+1}(E)=\int_{0}^{E} \frac{\omega(0,1, E-z) \omega(p, h-1, z)}{\omega(p, h, E)} \omega(1,2, E-z) d z$

where $z$ is the core energy.

The form of the particle-hole state density considered is that proposed by Oblozinsky [31]:

$$
\begin{aligned}
\omega(p, h, E)= & \frac{g^{N}}{p ! h !(N-1) !} \sum_{i=0}^{p} \sum_{k=0}^{h}(-1)^{i+k}\left(\begin{array}{l}
p \\
i
\end{array}\right)\left(\begin{array}{l}
h \\
k
\end{array}\right) \\
& \times \Theta\left(E-\alpha_{p h}-i B-k F\right) \\
& \times\left(E-A_{p h}-i B-k F\right)^{N-1}
\end{aligned}
$$

where $B$ and $F$ is the binding and Fermi energy, respectively; the step function $\Theta(x)$ is unity for $x>0$ and zero otherwise; $\alpha_{p h}$ is the minimum energy needed to excite $p$-particles and $h$-holes satisfying the Pauli principle and is given by

$$
\alpha_{p h}=\frac{1}{2}\left(\frac{p^{2}+p+h^{2}-h}{g}\right)
$$

and the quantity

$$
A_{p h}=\frac{1}{4}\left(\frac{p^{2}+p+h^{2}-3 h}{g}\right),
$$

accounts for Pauli blocking. In Ref. [28] the density of singleparticle(hole) states is derived from the equidistant singleparticle model, resulting in a n-stage $Y_{n}(E)$ function with a direct dependence on $E^{2}$. Therefore, for resonances at energies far away from the nucleon binding energy (as in isoscalar $G D R$ ) this outcome is not a good option because the spreading width involves only intermediate bound states. This way, we use the $Y_{n}(E)$ function proposed by Oblozinsky [31] where the level density is obtained restricting the nucleons to bound states, limiting the energy dependence.

After this brief discussion about the $F K K$ approach, we present some necessary modifications to connect it with the nuclear structure calculations like RPA. The definition of the spreading width in Eq.(1) and the form as the density of states was written in Eq.(2) permits to factor the width in terms of the product of the functions $X$ and $Y$ in Eq.(3), resulting a $X$ function that does not depend on the excitation energy $E$. Thus, the $X$ and $Y$ functions are calculated separately, resulting in a complete uncoupling between the angular momentum of the excited particle-hole pairs and the energy where they are considered in the calculation of the width. This way, all $1 p-1 h$ pairs are treated in the same foot in any energy, making the results to depend strongly on the particle-hole basis considered. On the other hand, in RPA approach, the excitation probability of a specific particle-hole pair depends on the excitation energy and its angular momentum coupling. Therefore, since we have accounted a fairly complete $1 p-1 h$ basis in a RPA calculation, the results do not undergo considerable alteration by including another more internal (or more external) single-particle level in the configuration basis, besides those already taken into account in the "fairly complete" basis. Nevertheless, as in the $F K K$ approach the constraint between the energy and angular momentum of the single-particle level is broken, the spreading width calculation does not take account these important microscopic informations about the occurrence probability of each pair $1 p-1 h$ on the energy of the $G R$.

In order to include these microscopic informations calculated by $R P A$, to minimize the dependence with the $1 p-1 h$ basis and the number of possible intermediate couplings, we have implemented some modifications in the original form [28] of the $X$ function. The main modification consists in to take into account the excitation probability of each $1 p-1 h$ pair that is accessed in the energy in which the calculations are performed (this proposal is hereafter referred to as $R P A+F K K$ approach). This reformulated $X$ function is given by:

$$
\begin{array}{r}
X_{n J}^{\downarrow(n+1)}(E)=2 \pi \sum_{j Q j_{4} j_{3}} P_{Q j_{4}}^{J} \frac{R_{1}(j) R_{1}(Q) R_{N-1}\left(j_{4}\right)}{R_{N}(J)} \\
\times \frac{\left(2 j_{3}+1\right)}{(2 Q+1)} \Delta\left(Q J j_{4}\right) \sum_{j_{1} j_{2}} \mathcal{P}_{j_{1} j_{2}}^{j_{3}} R_{1}\left(j_{1}\right) R_{1}\left(j_{2}\right) \\
\quad \times\left(\left\langle\left\{j_{1} j_{2}\right\}_{j_{3}}|V|\{Q j\}_{j_{3}}\right\rangle\right)^{2} L\left(E, E_{2}\right)
\end{array}
$$

where the inclusion of the factor $\mathcal{P}_{j_{p} j_{h}}^{J}$ controls the probability of accessing each $1 p-1 h$ configuration, and also it minimizes the dependences with the $1 p-1 h$ basis and the number of $j_{3}$ couplings. We have taken into account only phonons $\left(j_{3}\right)$ with energy smaller than the energy of particle threshold, as well as in the calculations based on the second $R P A$ versions [14-18]. The quantity $L\left(E, E_{2 p-2 h}\right)$ is a lorentzian type function,

$$
L\left(E, E_{2 p-2 h}\right)=\frac{\eta^{2}}{\left(E-E_{2 p-2 h}\right)^{2}+\eta^{2}},
$$


with $E_{2 p-2 h}=\varepsilon_{j}+\varepsilon_{j_{1}}-\varepsilon_{j_{4}}-\varepsilon_{j_{2}}$ ( $\varepsilon$ is the energy of the singleparticle level). It simulates the energy denominator of the second $R P A$ calculation and gives a measurement of the fragmentation of the RPA solution into $2 p-2 h$ space. Thus the accessibility of some single-particle level $j$ may be considered. We would like to emphasize that now the calculation of $X$ function is performed in each excitation energy $(E)$ at the $G R$ energy position, and with the respective $\mathcal{P}_{j_{p} j_{h}}^{J}$ and $L\left(E, E_{2 p-2 h}\right)$ factors.

We define the factors $\mathcal{P}_{j_{p} j_{h}}^{J}$ as function of the $1 p-1 h$ forward $R P A$ amplitudes $\left(x_{j_{p} j_{h}}^{J}\right)$ :

$$
\mathcal{P}_{j_{p} j_{h}}^{J}=\frac{N_{P}\left|x_{j_{p} j_{h}}^{J}\right|^{2}}{\sum_{j_{p} j_{h}\left(\Gamma_{p} \approx 0\right)}\left|x_{j_{p} j_{h}}^{J}\right|^{2}},
$$

where $N_{p}$ is the number of bound (or with single-particle width too smaller than $G R$ width) $1 p-1 h$ configurations coupling to $\vec{J}$. The $R P A$ amplitudes are obtained by a diagonalization of the complex equation:

$$
\left(\begin{array}{cc}
A & B \\
-B & -A
\end{array}\right)\left(\begin{array}{c}
x^{m} \\
y^{m}
\end{array}\right)=\widehat{\varepsilon}_{m}\left(\begin{array}{c}
x^{m} \\
y^{m}
\end{array}\right)
$$

where

$$
A_{p h p^{\prime} h^{\prime}}=\left(\widehat{\varepsilon}_{p}-\varepsilon_{h}\right) \delta_{p p^{\prime}} \delta_{h h^{\prime}}+V_{p h^{\prime} h p^{\prime}} ; B_{p h p^{\prime} h^{\prime}}=V_{p p^{\prime} h h^{\prime}}
$$

and $\widehat{\varepsilon}_{p}\left(\widehat{\varepsilon}_{p}=\varepsilon_{p}-\frac{1}{2} i \Gamma_{p}\right)$ are the complex energies of the single-particle resonances $[32,33]$. The real part of the complex eigenvalues $\widehat{\varepsilon}_{m}$ gives the excitation energy $\left(\varepsilon_{m}\right)$, and the imaginary part gives the escape width $\left(\Gamma_{m}^{\uparrow}\right)$ of the $1 p-1 h$ excitation mode (See Refs. [10] and [20] for more details).

The discrete single-particle energies were evaluated by solving the Schrödinger equation with Woods-Saxon potential, including the centrifugal and Coulomb (as a uniformly charged sphere) terms. The positive single-particle energy and its respective width were calculated in a projection technique to continuum discretization approach discussed in Refs.[32, $33]$. The $R P A$ calculation was done by utilizing the LandauMigdal residual interaction:

$$
\begin{aligned}
& V_{p h}\left(\overrightarrow{r_{1}}, \overrightarrow{r_{2}}\right)=C_{0}\left[f\left(r_{1}\right)+f^{\prime}\left(r_{1}\right) \overrightarrow{\tau_{1}} \cdot \overrightarrow{\tau_{2}}+\right. \\
& \left.+\overrightarrow{\sigma_{1}} \cdot \overrightarrow{\sigma_{2}}\left(g\left(r_{1}\right)+g^{\prime}\left(r_{1}\right) \overrightarrow{\tau_{1}} \cdot \overrightarrow{\tau_{2}}\right)\right] \delta\left(\overrightarrow{r_{1}}-\overrightarrow{r_{2}}\right)
\end{aligned}
$$

where $f, f^{\prime}, g$ and $g^{\prime}$ are dimensionless and density dependent parameters:

$$
F(r)=F^{e x}+\left(F^{i n}-F^{e x}\right) \xi(r) .
$$

The set of the interaction parameters was adjusted to eliminate the spurious state $1^{-}$(see TABLE I) and to reproduce the first $3^{-}$excited state for each nucleus under consideration. Since the neutron and proton densities are too different in nuclei with neutron excess, it is more appropriate to separate the nucleon density into neutron and proton parts, $\xi(r)=\frac{N}{A} \xi_{v}(r)+\frac{Z}{A} \xi_{\pi}(r)$, where each part is given by $\xi_{k}(r)=\frac{1}{1+e^{\left(r-R_{k}\right) / a_{k}}}$, with $k=v(\pi)$ for neutron (proton) [20].
In this same sense, the radial single-particle orbits are represented by harmonic oscillator radial wave functions with different size parameters for neutrons and protons, $b_{k}^{2} \approx$ $\frac{4}{(3)^{\frac{4}{3}}}\left\langle r^{2}\right\rangle_{k}\left(X_{k}\right)^{-\frac{1}{3}}$ [20], where $\left\langle r^{2}\right\rangle_{k} \approx \frac{3}{5} R_{k}^{2}+\frac{7}{5} \pi^{2} a_{k}^{2}$ [34].

We have adopted the following criterion in order to choose the parameter $\eta$ in Eq.(15):

$$
\eta=\eta_{m}=\min \left(\frac{\varepsilon_{m}-\varepsilon_{m-1}}{4}, \frac{\varepsilon_{m+1}-\varepsilon_{m}}{4}\right),
$$

where $\varepsilon_{m}$ are the energies obtained in $R P A$ calculation around the $G R$ position. We have considered only $R P A$ solutions $\left(\varepsilon_{m}\right)$ that exhaust at least $1 \%$ fraction of the the Energy Weighted Sum Rule $(E W S R)$. This criterion assure that we have considered only $2 p-2 h$ poles, $E_{2 p-2 h}$, in the neighborhood of solution $\varepsilon_{m}$, i.e., the common area among the lorentzian $L\left(\varepsilon_{m}, E_{2 p-2 h}\right)$ and its neighboring lorentzians, $L\left(\varepsilon_{m-1}, E_{2 p-2 h}\right)$ and $L\left(\varepsilon_{m+1}, E_{2 p-2 h}\right)$, is small. Then we have not taken account the $2 p-2 h$ poles that are lied out of the neighborhood of $\varepsilon_{m}$ (energy interval: $\left[\varepsilon_{m-1}+2 \eta, \varepsilon_{m+1}-2 \eta\right]$ ).

This way, various $R P A$ peaks can be superposing in the energy interval around $G R$, and the medium values of the energy and width are obtained through an average process over the involved peaks. These medium values are calculated by performing the weighted average:

$$
\langle a\rangle=\frac{\sum_{m} P_{m} a_{m}}{\sum_{m} P_{m}}
$$

where $a_{m}$ represents the value to be averaged and the weights, $P_{m}$, are the intensities of each peak relative to the fraction of $E W S R$ that they exhaust in the specific energy interval. For an arbitrary energy interval $\left[E_{I}, E_{F}\right]$ we get the definitions:

$$
P_{m}(\%) \equiv 100 \times \frac{\int_{E_{I}}^{E_{F}} S_{F}^{m}(E) E d E}{E W S R},
$$

where the strength function $S_{F}(E)$ is written as

$$
S_{F}(E)=\sum_{m} S_{F}^{m}(E)
$$

and

$$
E W S R=\int_{E_{I}}^{E_{F}} S_{F}(E) E d E
$$

In order to calculate the the strength function $S_{F}(E)$ we have assuned the particle-hole matrix element of the 1-body dipole operator $\left(\widehat{F}_{\lambda=1}\right)$ as

$$
\widehat{F}_{1 M}^{T=1}=e_{k} r Y_{1 M}
$$

for isovector $(T=1)$ electric dipole transition, where $e_{k}$ is the nucleon effective charge, i.e., $e_{v(\pi)}=-\frac{e Z}{A}\left(\frac{e N}{A}\right)$, and

$$
\widehat{F}_{1 M}^{T=0}=\left(r^{3}-\eta_{s} r\right) Y_{1 M}
$$

for isoscalar $(T=0)$ dipole transition, where $\eta_{s}=\frac{5}{3}\left\langle r^{2}\right\rangle$ to eliminate (reduce) the contribution of the spurious state mixing $[35,36]$. 
Another point that deserves a special attention is the part that deals with the residual interaction used in the calculation. The consistency of the $R P A+F K K$ approach is guaranteed by using the same Landau-Migdal interaction to diagonalize the $R P A$ equations and to compute the $X$ function. However, in the $F K K$ calculations [28, 30, 37, 38], the two-body interaction was assumed to be the simplest zero-range form

$$
V\left(\vec{r}_{1}, \vec{r}_{2}\right)=V_{0}\left(\frac{4}{3} \pi r_{0}^{3}\right) \delta\left(\vec{r}_{1}-\vec{r}_{2}\right),
$$

where the strength of the interaction, $V_{0}$, is a free parameter. It is frequently adjusted in order to reproduce some experimental data that can be calculated by the formalism. There is no standard procedure to adjust $V_{0}$, and it is controlled in each specific calculation. This makes possible many other different forms of getting such adjustments, what could cause great variations on the $V_{0}$ values. In this context, the studies performed by R. Bonetti and L. Colombo [37] have showed that $V_{0}$ increases when more realistic ingredients are included in the computation of matrix element of the interaction. In a series of calculations to reproduce the widths of precompound and compound nuclear $r$-stage, extracted from the experimental data about ${ }^{27} \mathrm{Al}\left({ }^{3} \mathrm{He}, p\right)$ reaction, they used different details concerning the interaction, wave functions and level densities, and they verified the occurrence of great variations in the $V_{0}$ values. In another way, distinct procedures were adopted to fit the experimental data in the Refs.[28, 30, 38] to treat different nuclear reactions. The varied forms to control the parameters of the residual interaction, as well as the management of $V_{0}$, seem to be a consequence of the difficulty to establish more general criteria to guide such adjustments.

In our proposal we have been adapting the $F K K$ formalism to be applied in consonance with the RPA approach. Proceeding this way, the parameters of the residual interaction had been adjusted at level of the RPA calculation in a standard way, in order to reproduce the excited states with the lowest energies and to eliminate the spurious solution at zero energy. Thus, the results for the $G R$ are obtained as consequence of this parametrization. Soon after, this same set of parameters was used to calculate the spreading width. This procedure is taken to keep the coherence between these two types of calculations for escape and spreading widths. In this sense, we do not have total freedom to choose the parameters, and we may interpret that the adjustments of a free parameter like $V_{0}$ can accommodate some details that should be part of the calculations. This statement becomes more evident when we write the squared matrix element of Eq.(14) in the following form:

$$
\begin{aligned}
\left(\left\langle j_{1} j_{2} ; j_{3}|\widetilde{V}| Q j ; j_{3}\right\rangle\right)^{2}= & \mathcal{P}_{Q j_{4}}^{J} \mathcal{P}_{j_{1} j_{2}}^{j_{3}}\left(\left\langle j_{1} j_{2} ; j_{3}|V| Q j ; j_{3}\right\rangle\right)^{2} \\
& \times L\left(\varepsilon_{m}, E_{2 p-2 h}\right)
\end{aligned}
$$

with $\widetilde{V}$ in the form given by the expression (28). Thus, the nuclear structure effects displayed by $\mathcal{P}_{Q j_{4}}^{J}, \mathcal{P}_{j_{1} j_{2}}^{j_{3}}$ and $L\left(\varepsilon_{m}, E_{2 p-2 h}\right)$ quantities could be hidden behind the adjustments of $V_{0}$.
TABLE I: Parameters of Landau-Migdal residual interaction.

\begin{tabular}{cccccccc}
\hline \hline $\mathrm{Nucl.} \mathrm{C}_{0}\left(\mathrm{MeVfm}^{3}\right)$ & $f^{\text {in }}$ & $f^{e x}$ & $f^{\text {in }}$ & $f^{\prime e x}$ & $g$ & $g^{\prime}$ \\
\hline${ }^{208} \mathrm{~Pb}$ & 368.75 & 0.200 & -1.474 & 1.5 & 1.5 & 0.635 & 0.70 \\
${ }^{48} \mathrm{Ca}$ & 300.0 & -0.002 & -1.282 & 0.76 & 2.30 & 0.05 & 0.94 \\
${ }^{60} \mathrm{Ca}$ & 300.0 & -0.002 & -2.100 & 0.76 & 2.30 & 0.51 & 0.70 \\
\hline \hline
\end{tabular}

TABLE II: Parameters of Fermi distribution and harmonic oscillator radial wavefunctions.

\begin{tabular}{ccccccc}
\hline \hline Nucl. & $R_{v}(\mathrm{fm})$ & $a_{v}(\mathrm{fm})$ & $R_{\pi}(\mathrm{fm})$ & $a_{\pi}(\mathrm{fm})$ & $b_{v}(\mathrm{fm})$ & $b_{\pi}(\mathrm{fm})$ \\
\hline${ }^{208} \mathrm{~Pb}$ & 6.84 & 0.50 & 6.60 & 0.50 & 2.41 & 2.51 \\
${ }^{48} \mathrm{Ca}$ & 3.34 & 0.65 & 3.05 & 0.65 & 1.95 & 1.97 \\
${ }^{60} \mathrm{Ca}$ & 4.34 & 0.60 & 3.68 & 0.60 & 2.09 & 2.11 \\
\hline \hline
\end{tabular}

\section{RESULTS AND DISCUSSIONS}

In this section we shall present the results for spreading widths for giant dipole resonances in ${ }^{208} \mathrm{~Pb}$ and ${ }^{48,60} \mathrm{Ca}$ nucleus. The set of parameters of residual interaction and harmonic oscillator radial wave function are given in TABLES I and II, respectively.

In the calculation of the spreading width we have taken into account the contributions of the intermediate phonons with multipole $j_{3} \leq 6$, natural parity and energy smaller than the neutron separation energy $S_{n}$. The features of these low-lying modes are obtained by the continuum RPA described in Ref [20]. This procedure is similar to others microscopic calculations which considering the coupling to low-lying phonons [14-18]. Our results for $G R$ widths are summarized in TABLE III. The comparison with the experimental data was showed a quite reasonable agreement.

The first application test refers to the spreading width calculation of the isovector $G D R$ in ${ }^{208} \mathrm{~Pb}$. The experimental data get this resonance located around $13.5 \mathrm{MeV}$ with a total width of $4.0 \mathrm{MeV}$ [39]. The decay of this resonance in heavy nucleus is broadly dominated by the statistical mechanism, being compatible with a small direct neutron branching ratio. Our calculation gets a medium energy around of $10.8 \mathrm{MeV}$ with a null escape width and a spreading width $\left(\left\langle\Gamma^{\downarrow}\right\rangle=4.4 \mathrm{MeV}\right)$ that fits the total width, indicating that the decay of isovector GDR in ${ }^{208} \mathrm{~Pb}$ is dominated by more complex mechanisms than the direct one, as it is expected for heavy nuclei. This result is in good accordance with the statistical calculations to analyze the neutron spcetra [40]. The centroid in energy is slightly shifted in relation to the experimental data, which might have been caused by the need of going besides $1 p-1 h$ excitations to explain the structure of this resonance and also by the fittings of Landau-Migdal residual interaction [41, 42].

On the other hand the results for the ISGDR are shown different, presenting a balanced competition between the direct and more complex decay modes. The experimental data about the isoscalar GDR are not well defined, and present some discrepancies among them. There are some discussions in the recent literature in relation to its correct location and their widths [22-24, 26]. Our calculations predict a considerable strength in the energy region above $20 \mathrm{MeV}$, which is com- 
TABLE III: Summarized results.

\begin{tabular}{|c|c|c|c|}
\hline Nucleus & Mode & $\left\langle\Gamma^{\uparrow}\right\rangle(\mathrm{MeV})$ & $\left\langle\Gamma^{\downarrow}\right\rangle(\mathrm{MeV})$ \\
\hline \multirow{2}{*}{${ }^{208} \mathrm{~Pb}$} & IVGDR & 0.00 & 4.4 \\
\hline & ISGDR & 2.67 & 3.3 \\
\hline${ }^{48} \mathrm{Ca}$ & IVGDR & 0.13 & 4.3 \\
\hline${ }^{60} \mathrm{Ca}$ & IVGDR & 0.56 & 0.0 \\
\hline \multicolumn{4}{|c|}{ Experimental values } \\
\hline & & $\langle E\rangle(\mathrm{MeV})$ & $\langle\Gamma\rangle(\mathrm{MeV})$ \\
\hline \multirow[t]{2}{*}{${ }^{208} \mathrm{~Pb}$} & IVGDR & 13.5 & $4.0[39]$ \\
\hline & ISGDR & $20-23$ & $2.5-10[22-24,26]$ \\
\hline${ }^{48} \mathrm{Ca}$ & IVGDR & 19.9 & $7.0[44]$ \\
\hline
\end{tabular}

posed by the presence of various narrow peaks superposing to exhaust about $82 \%$ of the $E W S R$ between $20-30 \mathrm{MeV}$. These peaks are mainly composed by $3 \hbar \omega$ transitions involving the neutrons and protons of the externals shells. The energy and escape width of this resonance were evaluated by performing a weighted average on the energies and widths of the peaks that compose it, the weights being the intensities of each peak relative to the $82 \%$ of the EWSR that they exhaust. The centroid was calculated at $24.4 \mathrm{MeV}$ with an average escape width of $2.7 \mathrm{MeV}$. Then, making use of these results for $I S G D R$ in ${ }^{208} \mathrm{~Pb}$, provided by the RPA calculations, we proceed the calculation of the spreading width and we obtained $\left\langle\Gamma^{\downarrow}\right\rangle=3.3 \mathrm{MeV}$, resulting a total width $\langle\Gamma\rangle=6.0 \mathrm{MeV}$. The result of our escape width is larger than the value of $1.9 \mathrm{MeV}$ encountered by another calculation of continuum RPA [43], while our calculated spreading width is in agreement with the fits from Ref.[26], and also, with the value of $3.2 \mathrm{MeV}$ used in the analysis of the Ref.[43].

The others results refer to the analyses of the decay mechanisms of the isovector giant dipole resonances in neutron-rich calcium isotopes, ${ }^{48} \mathrm{Ca}$ and ${ }^{60} \mathrm{Ca}$. Experimentally, the isovector $G D R$ in ${ }^{48} \mathrm{Ca}$ is localized around $19.5 \mathrm{MeV}$ with width of $7.0 \mathrm{MeV}$ [44]. We have found about $97 \%$ of the EWSR between 10 and $21 \mathrm{MeV}$ by performing our RPA calculation, with two peaks exhausting $47 \%$ of the $E W S R$ around $20 \mathrm{MeV}$. The mean energy is about $15.6 \mathrm{MeV}$ with a very small escape width: $\left\langle\Gamma^{\uparrow}\right\rangle \approx 130 \mathrm{keV}$, reflecting the narrow single-particles widths. This result is in disagreement with the Strauch et. al. estimates [45] which have given about $40 \%$ of direct neutron escape for GDR decay in this nucleus. This large fraction was deduced by comparison of the residual nucleus excitation spectrum $\left({ }^{47} \mathrm{Ca}\right)$, which was measured in ${ }^{48} \mathrm{Ca}\left(e, e^{\prime} n\right)$ reaction, with the statistical model calculations. On the contrary, our result for spreading width $\left(\left\langle\Gamma^{\downarrow}\right\rangle=4.3 \mathrm{MeV}\right)$ is in good agreement with the statistical analysis performed in this experimental work [45], which is compatible with $60 \%$ of the total width. With relation to ${ }^{60} \mathrm{Ca}$, the calculated spreading width in the isovector $G D R$ region, around $15 \mathrm{MeV}$, for this nucleus is very small, even considering many low-lying energy phonons. This fact reinforce the statement which the two or more neutron escape should be important for GDR decay in ${ }^{60} \mathrm{Ca}$ [20]. The spreading is small because there are few accessible intermediaries bound $1 p-1 h$ pairs.

The widths summarized in TABLE III can assist us to make a measure of the competition among the direct and statistical decay mechanisms, for so much, we define the direct decay branching ratio as $b^{\uparrow}=\Gamma^{\uparrow} / \Gamma$ and the statistical branching as $b^{\downarrow}=1-b^{\uparrow}$, where $\Gamma=\Gamma^{\uparrow}+\Gamma^{\downarrow}$ is the total width. The branching ratios should be understood as reference values that indicate the degree of fragmentation of the giant resonances. Thus, the null escape width for $I V G D R$ in ${ }^{208} \mathrm{~Pb}$ represents the largest domain of the statistical decay $\left(b^{\downarrow} \simeq 1\right)$ as it happens in heavy nuclei [40]. In a different way, the results for isoscalar $E 1$ in ${ }^{208} \mathrm{~Pb}$ indicate that this excitation mode should have a strong fragmentation of its microscopic structure in $1 p-1 h$ and $2 p-2 h$ components. The large direct branching ratio ( $b^{\uparrow} \simeq 0.45$ ) of the ISGDR in this same nucleus is composed by one neutron $\left(b_{v}^{\uparrow} \simeq 0.32\right)$ and one proton $\left(b_{\pi}^{\uparrow} \simeq 0.13\right)$ direct decay, being strongly dominated by neutrons emission. This result reflects the fact that this resonance is located in higher energy than the isovector GDR and far beyond the neutron threshold. Thus, the direct decay channel is quite favored by this high energy and it competes equally with the statistical mechanism, unlike of what it happens with the isovector one. In TABLE IV we present the estimates for the partial escape widths, and their respective branching ratios, for the isoscalar $E 1$ in ${ }^{208} P b$. To evaluate the partial escape widths $\Gamma_{h}^{\uparrow}$ we also weighed the single-particle widths on their occurrence probabilities $\left(\left|x_{p h}^{m}\right|^{2}\right)$ [20]. With relationship to the ${ }^{48} \mathrm{Ca}$ the results for spreading width is compatible with a statistical branching of $b^{\downarrow}=0.60$. This result is in good agreement with the neutrons spectra analysis of Ref. [45]. A more delicate analysis refers to the ${ }^{60} \mathrm{Ca}$ nucleus and there are no experimental data to compare. In this nucleus the isovector giant $E 1$ resonance is possibly located above of the threshold of multiple neutrons emission (the neutron and proton separation energies for ${ }^{60} \mathrm{Ca}$ are respectively: $S_{n} \approx 3.5 \mathrm{MeV}$ and $\left.S_{p} \approx 25 \mathrm{MeV}[46,47]\right)$. In previous calculations [20] we had obtained that this resonance was composed mainly for some bounded $1 p-1 h$ excitations of protons, and that the most external neutrons belonging to the neutron skin had contributed to compose the structure of the pygmy resonance. Consequently, the microscopic calculation involving only $1 p-1 h$ configurations do not get enough intensity to the neutrons access the continuum region, resulting a very narrow total width.

In this point, it is worthwhile to do a brief comment on the overtones of monopole (ISGMR2) and quadrupole isoscalar (ISGQR2) giant resonances discussed in Ref. [19], whose the high energies peaks were calculated in $32.1 \mathrm{MeV}$ and $30.5 \mathrm{MeV}$, respectively. Proceeding to show the applicability of our proposal we also obtained these high energies peaks around of $30.0 \mathrm{MeV}$ for both modes, exhausting about $75 \%$ of the EWSR for ISGMR2, and about $40 \%$ for $I S G Q R 2$. In these very high excitation energies, the resonances are very wide because of the complex nature of their $n p-n h$ structures, as well as the multiple nucleons in the continuum region, difficulting the prevision of the widths. However, worthwhile to present our estimates for the total widths of these resonances as: $14 \mathrm{MeV}$ for monopole and $6 \mathrm{MeV}$ for quadrupole overtones. In the TABLE V , these results are compared with others theoretical calculations and experimental data found in the 
TABLE IV: Partial escape widths for one neutron and one proton direct decay from $I S G D R$ in ${ }^{208} \mathrm{~Pb}$ nucleus. The neutron and proton direct branching ratios are composed by $\left(b^{\uparrow}\right)_{\nu}=0.32$ and $\left(b^{\uparrow}\right)_{\pi}=$ 0.13 , respectively. The partial branching ratios are shown in the column $b_{h}^{\uparrow}$ for each neutron and proton hole.

\begin{tabular}{lll}
\hline \hline \multicolumn{1}{c}{ hole } & $\Gamma_{h}^{\uparrow}(\mathrm{MeV})$ & $b_{h}^{\uparrow}=\Gamma_{h}^{\uparrow} / \Gamma$ \\
\hline$\left(3 p_{3 / 2}\right)_{v}$ & 0.01 & 0.002 \\
\hline$\left(2 f_{5 / 2}\right)_{v}$ & 0.03 & 0.005 \\
\hline$\left(2 f_{7 / 2}\right)_{v}$ & 0.58 & 0.097 \\
\hline$\left(1 h_{9 / 2}\right)_{v}$ & 0.47 & 0.078 \\
\hline$\left(2 d_{3 / 2}\right)_{v}$ & 0.19 & 0.032 \\
\hline$\left(2 d_{5 / 2}\right)_{v}$ & 0.16 & 0.027 \\
\hline$\left(1 g_{7 / 2}\right)_{v}$ & 0.25 & 0.042 \\
\hline$\left(1 g_{9 / 2}\right)_{v}$ & 0.22 & 0.037 \\
\hline$\left(2 d_{3 / 2}\right)_{\pi}$ & 0.07 & 0.012 \\
\hline$\left(1 h_{11 / 2}\right)_{\pi}$ & 0.19 & 0.032 \\
\hline$\left(2 d_{5 / 2}\right)_{\pi}$ & 0.13 & 0.022 \\
\hline$\left(1 g_{7 / 2}\right)_{\pi}$ & 0.23 & 0.038 \\
\hline$\left(1 g_{9 / 2}\right)_{\pi}$ & 0.09 & 0.015 \\
\hline$\left(2 p_{1 / 2}\right)_{\pi}$ & 0.01 & 0.002 \\
\hline$\left(1 f_{5 / 2}\right)_{\pi}$ & 0.01 & 0.002 \\
\hline$\left(2 p_{3 / 2}\right)_{\pi}$ & 0.01 & 0.002 \\
\hline$\left(1 f_{7 / 2}\right)_{\pi}$ & 0.02 & 0.003 \\
\hline \hline
\end{tabular}

TABLE V: The mean energy and total width (both in $\mathrm{MeV}$ ) for $I S G M R 2$ and $I S G Q R 2$ in ${ }^{208} \mathrm{~Pb}$.

\begin{tabular}{lccccc}
\hline \hline Mode & \multicolumn{5}{c}{ This Work Ref.[48] } \\
\hline ISGMR2.[19] & $\langle E\rangle$ & 29.5 & 33.7 & 32.1 & Exp. [26] \\
& $\langle\Gamma\rangle$ & 14 & - & - & - \\
\hline ISGQR2 & $\langle E\rangle$ & 31.5 & - & 30.5 & $26.9 \pm 0.7$ \\
& $\langle\Gamma\rangle$ & 6 & - & - & $6.0 \pm 1.3$ \\
\hline \hline
\end{tabular}

literature.

Concluding, we have presented a theoretical approach that improves the $F K K$ method to include microscopic ingredients, in order to calculate the spreading width of giant resonances. A important point is that the spreading width is calculated in connection with the RPA formalism. The residual interaction used in $F K K$ approach is the same that was adjusted in RPA calculation.

\section{ACKNOWLEDGMENTS}

This work was supported in part by Conselho Nacional de Desenvolvimento Científico e Tecnológico (CNPq), Brazil.
[1] G. Bertsch, P. F. Bortignon, and R. A. Broglia, Rev. Mod. Phys. 55, 287 (1983).

[2] S. Kamerdzhiev, J. Speth, and G. Tertychny, Physics Reports 393, 1 (2004).

[3] S. Shlomo and G. Bertsch, Nucl. Phys. A 243, 507 (1975).

[4] K. F. Liu and N. Van Giai, Phys. Lett. B 65, 23 (1976).

[5] R. de Haro, S. Krewald, and J. Speth, Nucl. Phys. A388, 265 (1982).

[6] Ph. Chomaz, N. Van Giai, and S. Stringari, Phys. Lett. B 189 , 375 (1987).

[7] N. Van Giai, P. F. Bortignon, F. Zardi, and R. A. Broglia, Phys. Lett. B 199, 155 (1987).

[8] A. F. R. de Toledo Piza, Rev. Bras. Fis. 17, 195 (1987).

[9] P. Curutchet, T. Vertse, and R. J. Liotta, Phys. Rev. C 39, 1020 (1989).

[10] N. Teruya, A.F.R. de Toledo Piza, and H. Dias, Nuclear Physics A 556, 157 (1993).

[11] C. Yannouleas, M. Dworzecka, and J. J. Griffin, Nuclear Physics A 379, 256 (1982); ibid. 397, 239 (1983).

[12] S. Drozdz, S. Nishizaki, J. Speth, and J. Wambach, Physics Reports 197, 1 (1990).

[13] K. Takayanagi, K. Shimizu, and A. Arima, Nucl. Phys. A 477, 205 (1988).

[14] J. S. Dehesa, S. Krewald, J. Speth, and A. Faessler, Phys. Rev. C 15, 1858 (1977).

[15] S. Kamerdzhiev, G. Tertychny, J. Speth, and J. Wambach, Nucl. Phys. A 577, 641 (1994); S. Kamerdzhiev, J. Speth, and G. Tertychny, ibid. 624, 328 (1997).

[16] N. D. Dang, T. Suzuki, and A. Arima, Phys. Rev. C 61, 064304 (2000); N. D. Dang, V. Kim Au, T. Suzuki, and A. Arima, ibid. 63, 044302 (2001).

[17] Denis Lacroix, Sakir Ayik, and Philippe Chomaz, Phys. Rev. C 63, 064305 (2001).
[18] G. Colo and P. F. Bortignon, Nucl. Phys. A 696, 427 (2001).

[19] M. L. Gorelik, I. V. Safonov, and M.H. Urin, Phys. Rev. C 69, 054322 (2004)

[20] T. N. Leite and N. Teruya, Eur. Phys. J. A 21, 369 (2004).

[21] T. N. Leite, N. Teruya, and H. Dias, to be published in Int. J. Mod. Phys. E.

[22] B. F. Davis, U. Garg, W. Reviol, M. N. Harakeh et. al., Phys. Rev. Lett. 79, 609 (1997).

[23] H. L. Clark, Y. -W. Lui, D. H. Youngblood, K. Bachtr et. al. Nucl. Phys. A 649, 57c (1999); H. L. Clark, Y. W. Lui, and D. H. Youngblood, Phys. Rev. C 63, 031301(R) (2001).

[24] M. Uchida, H. Sakaguchi, M. Itoh, M. Yosoi et. al., Physics Letters B 557, 12 (2003).

[25] M. Uchida, H. Sakaguchi, M. Itoh, M. Yosoi et. al., Phys. Rev. C 69, 051301(R) (2004).

[26] M. Hunyadi, A. M. van den Berg, N. Blasi, C. Boumer et. al., Physics Letters B 576, 253 (2003); Nucl. Phys. A 731, 49 (2004).

[27] U. Garg, Nucl. Phys. A731, 3 (2004).

[28] H. Feshbach, A. Kerman and S. Koonin, Annals of Physics 125, 429 (1980).

[29] T. N. Leite, and N. Teruya, Braz. J. Phys. 35, 829 (2005).

[30] R. Bonetti, M. B. Chadwick, P. E. Hodgson, B. V. Carlson, and M. S. Hussein, Physics Reports 202, 171 (1991).

[31] P. Oblozinsky, Nucl. Phys. A 453, 127 (1986).

[32] N. Teruya, A.F.R. de Toledo Piza, and H. Dias, Phys. Rev. C44, 537 (1991).

[33] T. N. Leite, N. Teruya, and H. Dias, Int. J. Mod. Phys. E 11, 469 (2002).

[34] L. L. Salcedo, E. Oset, M. J. Vicente-Vacas, and C. GarciaRecio, Nucl. Phys. A 484, 557 (1988).

[35] I. Hamamoto, H. Sagawa, and X. Z. Zhang, Phys. Rev. C 57, R1064 (1998). 
[36] S. Shlomo and A.I. Sanzhur, Phys. Rev. C65, 044310 (2002).

[37] R. Bonetti and L. Colombo, Phys. Rev. C28, 980 (1983).

[38] T. Kawano, Phys. Rev. C 59, 865 (1998).

[39] A. Veyssiere, H. Beil, R. Bergere, P. Carlos, and A. Lepretre, Nucl. Phys. A159, 561 (1970).

[40] N. Teruya, H. Dias and E. Wolynec, Phys. Rev. C37, 2121 (1988).

[41] B. Schwesinger and J. Wambach, Nucl. Phys. A 426, 253 (1984).

[42] S. Kamerdzhiev, J. Speth, G. Tertychny, and V. Tselyaev, Nucl. Phys. A 555, 90 (1993).

[43] M. L. Gorelik, S. Shlomo, and M. H. Urin, Phys. Rev. C 62,
044301 (2000)

[44] G. J. OKeefe, M. N. Thompson, Y. I. Assafiri, R. E. Pywell, and K. Shoda, Nucl. Phys. A649, 239 (1987)

[45] S. Strauch, Nucl. Phys. A 649, 85c-92c (1999); S. Strauch, P. von Neumann-Cosel, C. Rangacharyulu, A. Richter et. al., Phys. Rev. Lett. 85, 2913 (2000).

[46] S. Im and J. Meng, Phys. Rev. C61, 047302 (2000).

[47] I. Hamamoto, H. Sagawa, and X. Z. Zhang, Phys. Rev. C 64, 024313 (2001).

[48] S. Shlomo, V. M. Kolomietz, and B. K. Agraval, Phys. Rev. Phys. Rev. C68, 064301 (2003). 